\title{
Evaluation of surface dose outside the treatment area for five breast cancer irradiation modalities using thermo-luminescent dosimeters
}

\author{
Suraj P Khanal ${ }^{1}$, Zoubir Ouhib ${ }^{2}$, Rashmi K Benda ${ }^{2}$, Theodora Leventouri ${ }^{1}$ \\ ${ }^{I}$ Department of Physics, Medical Physics Program, Florida Atlantic University, Boca Raton, Florida, USA \\ ${ }^{2}$ Department of Radiation Oncology, Lynn Cancer Institute, Boca Raton Regional Hospital, Boca Raton, Florida, USA
}

Received October 20, 2014; Revised December 10, 2014; Accepted December 30, 2014; Published Online January 10, 2015

\section{Original Article}

\begin{abstract}
Purpose: To measure and compare the surface dose outside the treatment area at six different points of interest (POIs) for five different breast cancer radiation treatment modalities by using thermo-luminescent dosimeters (TLDs). This experiment will evaluate the magnitude of the dose due to scatter and leakage radiation at different areas outside the target on a patient that could potentially lead, in the long term, to radiation induced secondary malignancies. Methods: TLD-100 were calibrated according to the University of Wisconsin Radiation Calibration Laboratory protocol and then used for dose measurements at selected POIs namely sternum, lower abdomen, contralateral breast, thyroid, shoulder, and eye. Twenty five breast cancer patients and the following modalities were included in this study: Strut-adjusted volume implant (SAVI), mammosite multi-lumen (ML), Accuboost, electron boost and photon boost. The surface doses in all patients were measured in a single fraction. The delivered target doses were normalized to $200 \mathrm{cGy}$. Finally, breast quadrant analysis was performed. Results: The maximum average dose for each POI was as follows: Sternum $6.51 \mathrm{cGy}$ (SD 2.93), lower abdomen $4.50 \mathrm{cGy}$ (SD 2.63), contralateral breast $8.52 \mathrm{cGy}$ (SD 3.86), thyroid $5.50 \mathrm{cGy}$ (SD 2.75), shoulder $5.58 \mathrm{cGy}$ (SD 2.77), and eye $2.65 \mathrm{cGy}$ (SD 0.68). The highest POI dose of $15.84 \mathrm{cGy}$ was found in contralateral breast. Conclusion: The measured surface dose at each POI varies with the modality of treatment. The surface doses show a strong correlation to the tumor bed location in the breast quadrant. The SAVI, electron boost, and photon boost modalities had delivered smaller surface dose at POIs than the Accuboost and Mammosite ML modalities. While the measured doses fall within the low range, its significance in producing second malignancies would require a large cohort of patients and a longer follow up.
\end{abstract}

Keywords: TLDs; Absorbed Dose; Deterministic Effect; Boost; Induced Cancer

\section{Introduction}

Breast conservation surgery combined with radiation has become an accepted alternative to total mastectomy for selected patients with early-stage breast cancer because of its comparable overall survival rate and positive impact on quality of life. ${ }^{1}$ About $30 \%$ of U.S. women with breast cancer undergo breast conservation surgery with radiation therapy. ${ }^{2}$ It is reported that, irradiation of surrounding tissues during breast radiotherapy can cause development of secondary cancers within these tissues. ${ }^{3}$ Consequently, there is increasing concern regarding radiation-related secondary cancer risks in long term radiotherapy survivors and a need to evaluate cancer risks at high radiation doses. ${ }^{4}$

The doses at the points outside the treatment area are small compared to the target dose. Nonetheless, these doses are of clinical interest because they are given to large parts of the body and there is a potential for long term adverse effects. ${ }^{5}$ In addition, low doses of radiation also have potential to induce secondary cancers. ${ }^{6,7}$ Concern for risk of radiation-induced malignancy is growing with the increasing number of cancer patients and several publications have widely discussed the probability of secondary malignancies after primary radiation treatment. $^{8,}$ 9-19 Out-of-field doses due to radiation are responsible for affecting cataract formation, fetus, cardiac toxicity, infertility and hypothyroidism. ${ }^{5,20-25}$

The estimated threshold doses for the most radiosensitive tissues in humans are: $15 \mathrm{cGy}$ for temporary sterility of testes, 50-200 cGy for detectable opacity of lens and 50 cGy for depression of hematopoiesis. ${ }^{26}$ It is reported that about $22 \%$ of

Corresponding author: Suraj P Khanal; Department of Physics, Medical Physics Program, Florida Atlantic University, Boca Raton, Florida, USA.

Cite this article as: Khanal SP, Ouhib Z, Benda RK, Leventouri T. Evaluation of surface dose outside the treatment area for five breast cancer irradiation modalities using thermo-luminescent dosimeters. Int J Cancer Ther Oncol 2015; 3(1):030117. D0I: 10.14319/ijcto.0301.17

[A part of this research was presented at American Brachytherapy Society (ABS) Annual Meeting, which was held on April 3rd, 2014 in San Diego, CA. It was also presented at Florida Chapter AAPM Spring Meeting, which was held on 28th March, 2014 in Orlando, FL] 
secondary cancers are induced in regions more than $5 \mathrm{~cm}$ away from the irradiated volume. ${ }^{27}$ The latency period for secondary cancer development varies with tissue type. Overall, the median secondary tumor latency period is reported as 7.4 years. $^{28}$

The surrounding normal tissues outside the treated area inevitably receive some radiation dose, regardless of the treatment modality. Contributing factors include leakage from housing source, scatter from beam modifiers, and internal scatter from the patient. ${ }^{29}$ Accurate measurement of surface dose allows for estimation of the damage risk especially to those organs that are sensitive to relatively low doses of radiation. Several papers are published on the evaluation of peripheral and organ doses due to breast cancer radiotherapy; among those the in vivo and phantom measurements using thermo-luminescent dosimeters (TLDs), a computerized Monte Carlo (MC) technique using a mathematical phantom, and several commercial treatment planning software (TPS). ${ }^{30-33}$ Their results vary from method to method, i.e., the peripheral dose difference between TPS and MC was reported up to $70 \%,{ }^{34}$ while the mean difference between MC out-of-field doses and TLD measurements was found $11.4 \% \pm 5.9 \% .^{30}$ The percent difference between the TPS and TLD measurement skin doses was found in the range from $-15 \%$ to $44 \% .^{35}$

The peripheral doses at $20 \mathrm{~cm}$ away from the target area was found $2.52 \mathrm{cGy}$ and $2.07 \mathrm{cGy}$ using TLDs in LINAC and tomotherapy delivery correspondingly, for a planning target volume (PTV) dose of $200 \mathrm{cGy} .{ }^{36}$ However, the surface dose measurement outside the treatment area at several points of interest in a real patient using TLDs is limited.

There are several treatment modalities for breast cancer. This study includes SAVI, Mammosite ML, Accuboost, Electron boost, Photon boost. SAVI uses 6,8 or 10 peripheral source channels with one center channel and inserted in collapse form into the tumor cavity through a small incision in the breast. ${ }^{37,38}$ The catheters are then expanded to conform to the shape of the cavity and allow for precise delivery of radiation. MammoSite ML consists of a balloon catheter that is inserted into the lumpectomy then expanded, and radiation is delivered through a tiny seed attached to the catheter, irradiating the area surrounding the cavity. ${ }^{39,} 40$ Accuboost applies breast brachytherapy without invasive catheters.

Two parallel-opposed beams are directed into the breast maximizing the target dose uniformity and minimizing the dose to the skin and other healthy breast tissues. ${ }^{41}$ The Boost dose is radiation targeted at the tissue near the lumpectomy site; this tissue needs the extra dose, because it is thought to contain "pre-cancerous" cells, therefore it is most likely to develop reoccurrences. ${ }^{42,} 43$ The modalities for supplementary doses after lumpectomy are electrons and photon boosts.
Electron boost is the favored method of boost delivery, because electrons permit penetration of superficial tissues with limited radiation deeper into the lung, heart and other internal organs. Photon boost is encouraged only for deep seated tumors for better coverage with sparing of organ at risk. ${ }^{44}$

The aim of this study is to measure and compare the surface dose outside the irradiated volume in breast cancer patients at six different POIs in the proximity of critical structures for five different radiation treatment modalities: SAVI, Mammosite ML, Accuboost, Electron boost and Photon boost. Such study could be of interest to clinical investigation for the risk of late radiation effects in a breast cancer patient as a result of primary radiotherapy. The delivered doses for all modalities were normalized to $200 \mathrm{cGy}$ in order to compare the doses in the POIs. The effective dose equivalent is estimated for all modalities.

\section{Methods and Materials}

\section{Calibration of TLDs and Sorting}

The TLD-100 $\left(92.5 \%{ }^{7} \mathrm{LiF}+7.5 \%{ }^{6} \mathrm{LiF}\right)$ of size $3 \times 3 \times 0.89 \mathrm{~mm}^{3}$ detector was chosen because it is sensitive to electrons, photons and neutrons. The TLDs were calibrated according to the protocol of the University of Wisconsin. First, 500 TLD-100 chips were annealed at a $400^{\circ} \mathrm{C}$ oven for one hour in an aluminum tray in order to de-excite all the traps in the crystal and erase all residual doses. Then the TLDs were transferred in an acrylic holder with numbered positions and placed in an annealing oven at $80^{\circ} \mathrm{C}$ for 24 hours in order to redistribute traps to the desired single peak glow curve. Then they were cooled down to room temperature overnight. All the TLDs were irradiated by a Hopewell G10 ${ }^{137} \mathrm{Cs}$ irradiator in the UWRCL. The exposure of the ${ }^{137} \mathrm{Cs}$ was $500 \mathrm{mR}$. The TLDs were read by using a Harshaw-5500 automatic reader after 24 hours. The whole process was repeated three times, except for the $400^{\circ} \mathrm{C}$ annealing process to determine the individual sensitivities of the TLDs.

During the final sorting process, TLDs with a reading deviation greater than 3\% were ignored. Average readings more than $51 \mathrm{nC}$ and less than $36 \mathrm{nC}$ were also ignored in order to control the large variation of readings among the TLDs. The average deviation (stand/mean) was $1.41 \%$.

\section{Energy and dose dependence of TLDs}

The dose response of the TLD-100 material depends on the energy that it has been exposed to. ${ }^{45}$ In the dose measurements, the relative dose response depends on the energy that has been used in calibrating the dosimeters. TLD-100 chips were calibrated with a relatively high energy source $\left({ }^{137} \mathrm{Cs}\right.$, average energy $662 \mathrm{keV}$ ) so that the response of a megavoltage photon beam will be very close to the calibrated energy. The used TLD intrinsic energy conversion factor for ${ }^{192} \mathrm{Ir}$ to 
${ }^{137} \mathrm{Cs}$ was $1.041 \pm 0.018 .{ }^{35}$ For the electron beam of $6 \mathrm{MeV}$ to ${ }^{60} \mathrm{Co}$ the conversion factor was $0.965 \pm 0.013{ }^{46}$ and for ${ }^{60} \mathrm{Co}$ to ${ }^{137} \mathrm{Cs}$ was $0.960 .{ }^{47}$

The response of the TLDs is also dose dependent. For low doses, the response is linear for photons and electrons, but for higher doses $(>10 \mathrm{~Gy})$ the response is non-linear. ${ }^{48}$ In this study, the maximum dose delivered at the central axis of target volume during treatment was 340 cGy. Therefore, a linear model has been applied to normalize the doses.

\section{Patient selection}

Twenty five cases of breast cancer patients had been selected to evaluate the skin surface dose outside the treatment area at six different points for five different treatment modalities. The number of patients in each modality, planning target volume (PTV), patient's body mass index (BMI), prescribed dose and pertinent treatment planning software are given in the Table 1 . The patients were selected according to the policies and guidelines of the institutional review board (IRB) of the Boca Raton Regional Hospital, Boca Raton, FL. Patients' consent had been taken to participate in the study.

\section{Points of interest (POIs)}

The skin surface of sternum, lower abdomen, contralateral breast (CLB), thyroid, shoulder, and eye (superficial surface of eyelid) were chosen as POIs as shown in Figure 2. These POIs are considered clinically important because of their high radiation sensitivity.

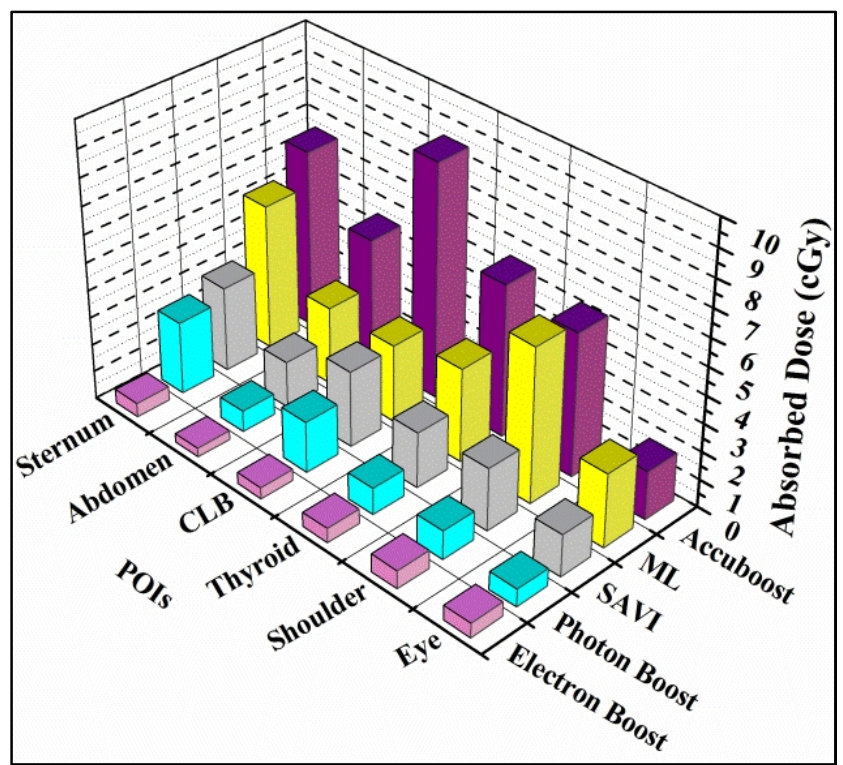

FIG. 1: The absorbed doses outside the treatment area at six POIs versus the treatment modalities. The delivered doses for all cases were normalized to $200 \mathrm{cGy}$.

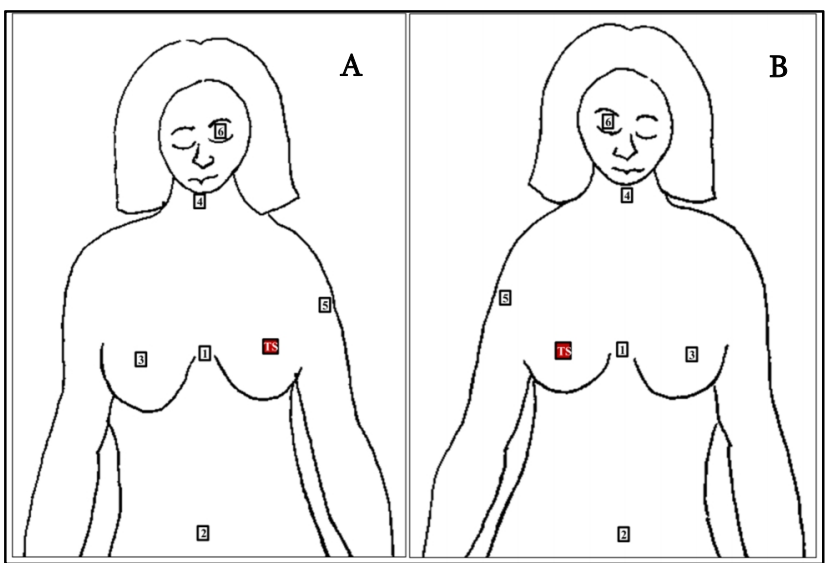

FIG. 2: A schematic diagram to show the points of interest (POIs). Six points were selected for TLDs placement. (1) Sternum; (2) Lower abdomen; (3) Contralateral breast (CLB); (4) Thyroid; (5) Shoulder; (6) Eye. Abbreviations: TS = Treatment site; $\mathrm{A}=$ Left breast treatment $\mathrm{B}=$ Right breast treatment.

\section{Method of study}

The calibrated TLDs were placed on the skin surface at six different POIs of the patients by the same person to minimize the placement errors of the chips. These points were selected such that the region of interest was most proximal to the tumor location. The TLDs were then exposed during patient treatment and read for evaluation. In each set of TLDs, two were held as control to account for shipping and transit dose. The absorbed dose was calculated by taking into account the background dose, an energy response factor, and a Cs-137 calibration factor using Equation (1).

$$
\begin{gathered}
\text { Absorbed dose }(c G y)=\frac{\text { reading }(n C)}{C s-137 \text { calibration factor }(n C / c G y) \times \text { energy response factor }} \\
-----(1)
\end{gathered}
$$

The patient's data were grouped under each modality and on tumor location in breast quadrant for comparison.

\section{Quadrant analysis}

Quadrant analysis was performed to investigate the relationship between the absorbed dose at the different POIs and the area of breast where the tumor is located. For this purpose, the breasts are divided into quadrants and named: Right upper outer quadrant (RUOQ), Right lower outer quadrant (RLOQ), Right upper inner quadrant (RUIQ), Right lower inner quadrant (RLIQ), Left upper outer quadrant (LUOQ), Left lower outer quadrant (LLOQ), Left upper inner quadrant (LUIQ) and Left lower inner quadrant (LLIQ). From the patients' records, it was found that the numbers of tumor location were: RUOQ (2), RLOQ (5), RUIQ (2), RLIQ (2), LUOQ (4), LLOQ (3), LUIQ(2) and LLIQ (1). The records of 4 out of 25 patients did not include the quadrant information. 
TABLE 1: The studied modalities with patients and treatment planning information.

\begin{tabular}{|c|c|c|c|c|c|c|}
\hline Modality & $\begin{array}{c}\text { Number } \\
\text { of patient }\end{array}$ & $\begin{array}{l}\text { PTV volume } \\
\text { (cc) }\end{array}$ & $\begin{array}{l}\text { Skin to tumor } \\
\text { distance }(\mathrm{mm})\end{array}$ & $\begin{array}{l}\text { Patient's } \\
\text { BMI }\end{array}$ & $\begin{array}{c}\text { Prescribed dose/fx } \\
(\mathrm{cGy})\end{array}$ & TPS \\
\hline Mammosite ML & 4 & $26-60$ & $8-12$ & $21-29$ & 340 & Oncentra \\
\hline Accuboost & 7 & $29-80$ & $9-15$ & $20-25$ & $180-200$ & Nucletron-ART \\
\hline SAVI & 6 & $20-66$ & $6-10$ & $24-50$ & 340 & Oncentra \\
\hline Electron Boost & 4 & $54-150$ & $9-14$ & $20-32$ & 200 & Eclipse \\
\hline Photon Boost & 4 & $55-174$ & $6-14$ & $22-32$ & 200 & Eclipse \\
\hline
\end{tabular}

Abbreviations: PTV = Planning target volume; BMI = Body mass index; TPS = Treatment planning software

TABLE 2: The averaged absorbed dose in centigray (cGy) outside the treatment area at six different POIs for the five studied modalities. The standard deviations are given in parenthesis.

\begin{tabular}{l|ccccc}
\hline \hline \multirow{2}{*}{ POI } & \multicolumn{5}{c}{ Absorbed dose in cGy for the studied modalities } \\
\cline { 2 - 6 } & Accuboost & SAVI & ML & $\begin{array}{c}\text { Electron } \\
\text { Boost }\end{array}$ & $\begin{array}{c}\text { Photon } \\
\text { Boost }\end{array}$ \\
\hline Sternum & $6.51(2.93)$ & $3.06(1.28)$ & $5.27(2.12)$ & $0.52(0.20)$ & $2.62(1.82)$ \\
Abdomen & $4.50(2.64)$ & $1.67(1.22)$ & $2.81(2.26)$ & $0.32(0.18)$ & $0.76(0.11)$ \\
CLB & $8.52(3.87)$ & $2.74(1.49)$ & $2.80(1.22)$ & $0.41(0.24)$ & $1.84(0.69)$ \\
Thyroid & $5.50(2.75)$ & $2.00(0.73)$ & $3.38(1.03)$ & $0.52(0.13)$ & $0.96(0.55)$ \\
Shoulder & $5.18(2.21)$ & $2.26(1.11)$ & $5.58(2.77)$ & $0.66(0.16)$ & $1.02(0.42)$ \\
Eye & $1.74(0.84)$ & $1.51(0.52)$ & $2.64(0.69)$ & $0.52(0.29)$ & $0.64(0.20)$ \\
\hline \hline
\end{tabular}

\section{Results}

The results of the out-of-field surface dose measurements on these 25 breast cancer patients for five different treatment modalities are listed in Table 2 and plotted in Figure 1. The absorbed doses in each POI were averaged for each modality group. As Figure 1 shows, the highest average skin surface dose was measured at contralateral breast treated with Accuboost modality. The high standard deviations in Table 2 are related to the number and specifics of patients in each modality.

From the quadrant analysis of the data in Table 3, it was found that 10 patients had cancer at the left breast and 11 patients had cancer at the right breast while about $67 \%$ of patients had a tumor in the outer quadrant. As Table 3 shows, four out of six patients having a tumor in the inner quadrant were treated with Accuboost. The ones with tumor in the inner upper quadrant had received higher doses to the thyroid, contralateral breast and the sternum. In patients with tumor in the left outer upper quadrant, the shoulder had received the highest dose. Relatively higher skin surface doses have been measured at the lower abdomen, contralateral breast and the sternum compared to the other POIs for patients treated with a tumor in the lower inner quadrants.

From all the modalities and patients studied, the maximum surface doses vary at the POIs with values: $15.84 \mathrm{cGy}(7.92 \%)$ in contralateral breast, $12.66 \mathrm{cGy}(6.33 \%)$ in sternum, 11.50 cGy (5.75\%) in thyroid, 11.13 cGy (5.56\%) in shoulder, 3.86 cGy (1.93\%) in eye and 8.6 cGy (4.30\%) in lower abdomen.
The numbers in parentheses are the corresponding percentages of the normalized delivered dose.

TABLE 3: Quadrant analysis result from the twenty one patients treated with different modalities. Tumor bed locations in the breast and corresponding maximum absorbed dose to the POIs are also given.

\begin{tabular}{|c|c|c|}
\hline Modalities & $\begin{array}{l}\text { Number of patients treated } \\
\text { (tumor bed location) }\end{array}$ & $\begin{array}{c}\text { POIs with maximum } \\
\text { dose }\end{array}$ \\
\hline \multirow{5}{*}{$\mathrm{AB}$} & $1(\mathrm{RLIQ})$ & CLB (6.41 cGy) \\
\hline & 1 (RUIQ) & Thyroid (11.50 cGy) \\
\hline & 1 (LUOQ) & Shoulder (8.25 cGy) \\
\hline & 1 (LUIQ) & CLB (15.84 cGy) \\
\hline & 1 (LLIQ) & Sternum (12.66 cGy) \\
\hline \multirow{3}{*}{ ML } & 2 (RUOQ) & Shoulder (11.13) \\
\hline & 1 (LUIQ) & Sternum (3.72 cGy) \\
\hline & 1 (RLOQ) & Sternum (5.64 cGy) \\
\hline \multirow{3}{*}{ SV } & 3 (RLOQ) & $\begin{array}{l}\text { Sternum (3.10 cGy), } \\
\text { Shoulder (4.19 cGy) }\end{array}$ \\
\hline & 2 (LUOQ) & Sternum (2.75 cGy) \\
\hline & 1 (LLOQ) & Sternum (5.26 cGy) \\
\hline \multirow{4}{*}{$\mathrm{PB}$} & 1 (RUIQ) & Sternum (5.23 cGy) \\
\hline & 1 (RLOQ) & Sternum (1.12 cGy) \\
\hline & 1 (RLIQ) & Sternum (2.46 cGy) \\
\hline & 1 (LLOQ) & CLB (2.37 cGy) \\
\hline \multirow{2}{*}{$\mathrm{EB}$} & 1 (LLOQ) & Shoulder $(0.78 \mathrm{cGy})$ \\
\hline & 1 (LUOQ) & Eye (0.98 cGy) \\
\hline
\end{tabular}

Abbreviations: $\mathrm{AB}=$ Accuboost; $\mathrm{ML}=$ Mammosite multi lumen; $\mathrm{SV}=\mathrm{SAVI} ; \mathrm{PB}=$ Photon boost $; \mathrm{EB}=$ Electron boost 
TABLE 4: The two way analysis of variance (ANOVA) test to compare the treatment modalities in terms of measured surface doses at POIs.

\begin{tabular}{|c|c|c|c|c|c|c|c|c|c|c|}
\hline Modalities & $\begin{array}{c}\mathrm{AB} \text { vs } \\
\mathrm{SV}\end{array}$ & $\begin{array}{c}\text { AB vs } \\
\text { ML }\end{array}$ & $\begin{array}{c}\mathrm{AB} \text { vs } \\
\mathrm{EB}\end{array}$ & $\begin{array}{c}\mathrm{AB} \text { vs } \\
\mathrm{PB}\end{array}$ & $\begin{array}{c}\text { SV vs } \\
\text { ML }\end{array}$ & $\begin{array}{c}\text { SV vs } \\
\text { EB }\end{array}$ & $\begin{array}{c}\text { SV vs } \\
\text { PB }\end{array}$ & $\begin{array}{c}\text { ML vs } \\
\text { EB }\end{array}$ & $\begin{array}{c}\text { ML vs } \\
\text { PB }\end{array}$ & $E B$ vs $P B$ \\
\hline$p$-value & 0.008 & 0.16 & 0.003 & 0.003 & 0.02 & 9E-04 & $4 \mathrm{E}-04$ & 0.001 & 0.004 & 0.049 \\
\hline
\end{tabular}

The two way analysis of variance (ANOVA) test was performed to compare the average doses at POIs for the five different treatment modalities. Results of the test are given in Table 4. It can be seen that Accuboost and Mammosite multi-lumen are not significantly different $(p=0.16$, at $5 \%$ significant level). SAVI values are significantly different from these two modalities ( $\mathrm{AB}$ and $\mathrm{ML}$ ) with $p<0.05$. The out-of-field skin surface doses measured with APBI (AB, SV and $\mathrm{ML}$ ) modalities are significantly different compared to electron boosts or photon boosts $(p<0.05)$. In addition the p-value for EB and PB are close to the significant level of 5\%.

The effective dose $\left(\mathrm{HE}_{\mathrm{E}}=\Sigma \mathrm{W}_{\mathrm{T}} \mathrm{H}_{\mathrm{T}}\right)$ provides a number that is proportional to the radiobiological adverse effect from an inhomogeneous type of radiation exposure. It has been defined and introduced by ICRP for risk management purposes. According to the ICRP (2007), the tissue specific weighting factors (Wт) for thyroid, breast, stomach, lungs, gonads and remainder tissues are $0.04,0.12,0.12,0.08$ and 0.12 respectively. Considering the closest tissues/organs from the skin surface, the calculated effective doses for the studied modalities are: Accuboost 3.45 rem, SAVI 1.45 rem, ML 2.46 rem, electron boost 0.32 rem and photon boost 0.87 rem. The beam quality factor for the photon and electrons are taken unity. The neutron generation term is ignored because of the low photon energy (less than $6 \mathrm{MV}$ ).

\section{Discussion}

This study shows that the measured absorbed doses at POIs treated with the same modality display large variations. The highest variation was found for the contralateral breast site treated with the Accuboost modality with a range from 2.67 cGy to $15.84 \mathrm{cGy}$. The tumors were located in the left upper outer quadrant (LUOQ) and the left upper inner quadrant (LUIQ) correspondingly. The second major variation was found in the shoulder treated with Mammosite ML with the dose range between $2.52 \mathrm{cGy}$ and $11.13 \mathrm{cGy}$. The tumors were located in the left upper inner quadrant (LUIQ) and the right upper outer quadrant (RUOQ) respectively.

The location of the tumor in the breast quadrant and distance of POIs from the treatment site are the two major factors influencing the dose to the POI, as expected from the inverse square law. Patient's BMI, size of tumor, size of breast, duration of treatment, orientation of field, patients' set up, applicators and catheters used during treatment could be additional affecting factors in receiving different skin surface doses at different POIs.
In the case of electron boosts, the measured out-of-field surface doses were small in all POIs due to predominantly ionizing events with atomic electrons of the tissue, resulting to absorbance of the incident electrons' energy within a few millimeters. However, in order to accurately justify the absorbed dose results with electron boost, calibration of TLDs has to be done with an electron beam, whereas the TLDs of the present study were calibrated with a photon beam.

\section{Conclusion}

The measured surface dose at each POI varies with the modalities of treatment. The surface doses show a strong correlation to the tumor bed location in the breast quadrant. The SAVI, electron boost, and photon boost had delivered smaller surface doses at POIs than the Accuboost and Mammosite ML modalities. While these doses are found within the low range, a longer follow up and a large cohort of patients could provide valuable information regarding the radiation induced secondary malignancies. It should be reminded that special care should be given to delivery parameters such as patient set up, transfer tube orientation, applicator orientation, and field set up in order to minimize the surface dose.

\section{Conflict of interest}

The authors declare that they have no conflicts of interest. The authors alone are responsible for the content and writing of the paper.

\section{Acknowledgements}

The first author would like to thank Larry A DeWerd, Ph.D., John A Micka, Clifford G Hammer and Keith A Kunugi for their guidance in TLDs calibration and support while at the University of Wisconsin-Madison. We also thank Vindu Kathriarachchi and Casey Curley for their comments on this manuscript.

\section{References}

1. Spear SL, Prada CA. Reduction mammaplasty in conjunction with breast conservation. Semin Plast Surg 2004; 18:255-60.

2. American Cancer Society Intermural research. Cancer treatment and survivorship facts and figures 2012-2013. 
3. Harvey EB, Brinton LA. Second cancer following cancer of the breast in Connecticut, 1935-82. Natl Cancer Inst Monogr 1985; 68:99-112.

4. Schneider U. Modeling the Risk of Secondary Malignancies after Radiotherapy. Genes 2011; 2:1033-49.

5. Taylor ML, Kron T. Consideration of the radiation dose delivered away from the treatment field to patients in radiotherapy. JMed Phys 2011; 36:59-71.

6. International Commission for Radiation Protection. Low-dose extrapolation of radiation-related cancer risk. ICRP Publication 99; 2005.

7. Committee on the biological effects of ionizing radiation. Health risks from exposure to low levels of ionizing radiation. Washington DC, National Research Council, National Academy Press; 2006.

8. Brown LM, Chen BE, Pfeiffer RM, et al. Risk of second non-hematological malignancies among 376,825 breast cancer survivors. Breast Cancer Res Treat 2007; 106:439-51.

9. Suit H, Goldberg S, Niemierko A, et al. Secondary carcinogenesis in patients treated with radiation: a review of data on radiation-induced cancers in human, non-human primate, canine and rodent subjects. Radiat Res 2007; 167:12-42.

10. Travis LB, Ng AK, Allan JM, et al. Second malignant neoplasms and cardiovascular disease following radiotherapy. JNCI J Natl Cancer Inst 2012; 104:357-70.

11. Boice JD Jr, Day NE, Andersen A, et al. Second cancers following radiation treatment for cervical cancer. An international collaboration among cancer registries. J Natl Cancer Inst 1985; 74:955-75.

12. Raymond JS, Hogue CJ. Multiple primary tumors in women following breast cancer, 1973-2000. Br J Cancer 2006; 94:1745-50.

13. Roychoudhuri R, Evans H, Robinson D, Møller H. Radiation-induced malignancies following radiotherapy for breast cancer. Br J Cancer 2004; 91:868-72.

14. Brenner DJ, Curtis RE, Hall EJ, Ron E. Second malignancies in prostate carcinoma patients after radiotherapy compared with surgery. Cancer 2000; 88:398-406.

15. Neugut AI, Robinson E, Lee WC, et al. Lung cancer after radiation therapy for breast cancer. Cancer 1993; 71:3054-7.

16. Ron E. Cancer risks from medical radiation. Health Phys 2003; 85:47-59.

17. Galper S, Gelman R, Recht A, et al. Second nonbreast malignancies after conservative surgery and radiation therapy for early-stage breast cancer. Int $J$ Radiat Oncol Biol Phys 2002; 52:406-14.

18. Fowble B, Hanlon A, Freedman G, et al. Second cancers after conservative surgery and radiation for stages I-II breast cancer: identifying a subset of women at increased risk. Int J Radiat Oncol Biol Phys 2001; 51:679-90.

19. Mellemkjaer L, Friis S, Olsen JH, et al. Risk of second cancer among women with breast cancer. Int J Cancer 2006; 118:2285-92.

20. van der Giessen PH, Hurkmans CW. Calculation and measurement of the dose to points outside the primary beam for CO-60 gamma radiation. Int J Radiat Oncol Biol Phys 1993; 27:717-24.

21. Doll R, Wakeford R. Risk of childhood cancer from fetal irradiation. Br J Radiol 1997; 70:130-9.

22. Sandeman TF. The effects of $x$ irradiation on male human fertility. BrJ Radiol 1966; 39:901-7.

23. Lee N, Chuang C, Quivey JM, et al. Skin toxicity due to intensity-modulated radiotherapy for head-and-neck carcinoma. Int J Radiat Oncol Biol Phys 2002; 53:630-7.

24. Benedick AF, Jan Van DG. Peripheral dose from megavoltage beams. Med Phys 1983; 10:809-17.

25. Jereczek-Fossa BA, Alterio D, Jassem J, et al. Radiotherapy-induced thyroid disorders. Cancer Treat Rev 2004; 30:369-84.

26. International Commission on Radiological Protection. Nonstochastic Effects of Ionizing Radiation. ICRP Publication 41 Ann ICRP 1984; 14.

27. Diallo I, Haddy N, Adjadj E, et al. Frequency distribution of second solid cancer locations in relation to the irradiated volume among 115 patients treated for childhood cancer. Int J Radiat Oncol Biol Phys 2009; 74:876-83.

28. Welte B, Suhr P, Bottke D, et al. Second malignancies in high-dose areas of previous tumor radiotherapy. Strahlenther Onkol 2010; 186:174-9.

29. Stovall M, Blackwell CR, Cundiff J, et al. Fetal dose from radiotherapy with photon beams: report of AAPM Radiation Therapy Committee Task Group No. 36. Med Phys 1995; 22:63-82.

30. Berris T, Mazonakis M, Stratakis J, et al. Calculation of organ doses from breast cancer radiotherapy: a Monte Carlo study. J Appl Clin Med Phys 2013; 14:4029.

31. Stovall M, Smith SA, Langholz BM, et al. Dose to the contra-lateral breast from radiotherapy and risk of second primary breast cancer in the WECARE study. Int J Radiat Oncol Biol Phys 2008; 72:1021-30.

32. Harrison RM, Wilkinson M, Rawlings DJ, Moore $M$. Doses to critical organs following radiotherapy and concomitant imaging of the larynx and breast. BrJ Radiol 2007; 80:989-95.

33. Berrington de GA, Curtis RE, Gilbert E, et al. Second solid cancers after radiotherapy for breast cancer in SEER cancer patients. Br J Cancer 2010; 102:220-26.

34. Joosten A, Matzinger O, Jeanneret-Sozzi W, et al. Evaluation of organ-specific peripheral doses after 
2-dimensional, 3-dimensional and hybrid intensity modulated radiation therapy for breast cancer based on Monte Carlo and convolution/ superposition algorithms: implications for secondary cancer risk assessment. Radiother Oncol 2013; 106:33-41.

35. Raffi JA, Davis SD, Hammer CG, et al. Determination of exit skin dose for 192Ir intracavitary accelerated partial breast irradiation with thermoluminescent dosimeters. Med Phys 2010; 37: 2693-702.

36. Kinhikar R, Gamre P, Tambe C, et al Peripheral dose measurements with diode and thermoluminescence dosimeters for intensity modulated radiotherapy delivered with conventional and un-conventional linear accelerator. JMed Phys 2013; 38:4-8.

37. Manoharan SR, Rodriguez RR, Bobba VS, et al. Dosimetry evaluation of SAVI-based HDR brachytherapy for partial breast irradiation. JMed Phys 2010; 35:131-6.

38. Bloom ES, Kirsner S, Mason BE, et al. Accelerated partial breast irradiation using the strut-adjusted volume implant single-entry hybrid catheter in brachytherapy for breast cancer in the setting of breast augmentation. Brachytherapy 2011; 10:178-83.

39. Dickler A, Kirk M, Choo J, et al. Treatment volume and dose optimization of MammoSite breast brachytherapy applicator. Int J Radiat Oncol Biol Phys 2004; 59:469-74.

40. Hepel JT, Wazer DE. A comparison of brachytherapy techniques for partial breast irradiation. Brachytherapy 2012; 11:163-75.

41. Rivard MJ, Melhus CS, Wazer DE, Bricault RJ Jr. Dosimetric characterization of round HDR 192Ir
Accuboost applicators for breast brachytherapy. Med Phys 2009; 36:5027-32.

42. Romestaig P, Lehingue $\mathrm{Y}$, Carrie C, et al. Role of a $10-\mathrm{Gy}$ boost in the conservative treatment of early breast cancer: results of a randomized clinical trial in Lyon, France. J Clin Oncol 1997; 15:963-8.

43. Kovacs A, Hadjiev J, Lakosi F, et al. Comparison of photon with electron boost in treatment of early stage breast cancer. Pathol Oncol Res 2008; 14:193-7.

44. Toscas JI, Linero D, Rubio I, et al. Boosting the tumor bed from deep-seated tumors in early-stage breast cancer: a planning study between electron, photon, and proton beams. Radiother Oncol 2010; 96:192-8.

45. Nunn AA, Davis SD, Micka JA, DeWerd LA. LiF:Mg,Ti TLD response as a function of photon energy for moderately filtered $\mathrm{x}$-ray spectra in the range of $20-250 \mathrm{kVp}$ relative to 60Co. Med Phys 2008; 35:1859-69.

46. Mobit PN, Nahum AE, Mayles P. The energy correction factor of LiF thermoluminescent dosemeters in megavoltage electron beams: Monte Carlo simulations and experiments. Phys Med Biol 1996; 41:979-93.

47. Maiello ML, Gulbin JF, Planque GD, Heaton HT. International Mini-intercomparision investigating an under response of TLDs calibrated with 137Cs. Radiat Prot Dosimetry 1990; 34:175-8.

48. McKinlay AF. Thermo-luminescence dosimetry medical physics handbooks 5, Adam Hilger Ltd; 1981:34-35. 